\title{
Keyphrase Generation for Scientific Document Retrieval
}

\author{
Florian Boudin $^{1}$, Ygor Gallina ${ }^{1}$, Akiko Aizawa ${ }^{2}$ \\ ${ }^{1}$ LS2N, Université de Nantes, France first.last@univ-nantes.fr \\ ${ }^{2}$ National Institute of Informatics, Tokyo, Japan aizawa@nii.ac.jp
}

\begin{abstract}
Sequence-to-sequence models have lead to significant progress in keyphrase generation, but it remains unknown whether they are reliable enough to be beneficial for document retrieval. This study provides empirical evidence that such models can significantly improve retrieval performance, and introduces a new extrinsic evaluation framework that allows for a better understanding of the limitations of keyphrase generation models. Using this framework, we point out and discuss the difficulties encountered with supplementing documents with -not present in textkeyphrases, and generalizing models across domains. Our code is available at https:// github.com/boudinfl/ir-using-kg.
\end{abstract}

\section{Introduction}

With the exponential growth of the scientific literature (Bornmann and Mutz, 2015), retrieving relevant scientific papers becomes increasingly difficult. Keywords, also referred to as keyphrases, provide an effective way to supplement paper indexing and improve retrieval effectiveness in scientific digital libraries (Barker et al., 1972; Zhai, 1997; Gutwin et al., 1999; Lu and Kipp, 2014). However, only few documents have assigned keyphrases, and those who do were, for the most part, selflabeled by their authors, thus exhibiting annotation inconsistencies (Strader, 2011; Suzuki et al., 2011). This has motivated an active line of research on automatic keyphrase extraction (see Hasan and $\mathrm{Ng}$ (2014) for an overview) and, more recently, keyphrase generation (Meng et al., 2017), where the task is to find a set of words and phrases that represents the main content of a document.

Although models for predicting keyphrases have been extensively evaluated on their ability to reproduce author's keywords, it still remains unclear whether they can be usefully applied in information retrieval. One reason for this lack of evidence may have been their relatively low performance discouraging attempts at using them for indexing (Liu et al., 2010; Hasan and $\mathrm{Ng}$, 2014). Yet, recently proposed models not only achieve much better performance, but also display a property that may have a significant impact on retrieval effectiveness: the capacity to generate keyphrases that do not appear in the source text. These absent keyphrases do not just highlight the topics that are most relevant, but provide some form of semantic expansion by adding new content (e.g. synonyms, semantically related terms) to the index (Greulich, 2011). The goal of this paper is two-fold: to gather empirical evidence as to whether current keyphrase generation models are good enough to improve scientific document retrieval, and to gain further insights into the performance of these models from an extrinsic perspective. Our contributions are listed as follows:

- We report significant improvements for strong retrieval models on a standard benchmark collection, showing that keyphrases produced by state-of-the-art models are consistently helpful for document retrieval, even, to our surprise, when author keywords are provided.

- We introduce a new extrinsic evaluation framework for keyphrase generation that allows for a deeper understanding of the limitations of current models. Using it, we discuss the difficulties associated with domain generalization and absent keyphrase prediction.

\section{Methodology}

This section presents our methodology for assessing the usefulness $(\$ 2.3)$ of keyphrase generation (§2.2) in scientific document retrieval (\$2.1).

\subsection{Scientific Document Retrieval}

Here, we focus on the task of searching through a collection of scientific papers for relevant docu- 
ments. All of our experiments are conducted on the NTCIR-2 test collection (Kando, 2001) which is, to our knowledge, the only available benchmark dataset for that task. It contains 322,058 documents ${ }^{1}$ (title and abstract pairs) and 49 search topics (queries) with relevance judgments. Most of the documents (98.6\%) include author keywords (4.8 per doc. on avg.), which we later use to investigate the performance of keyphrase generation models.

Documents cover a broad range of domains from pure science to social sciences and humanities, although half of the documents are about engineering and computer science. Queries are also categorized into one or more research fields (e.g. science, chemistry, engineering), the original intent being to help retrieval models in narrowing down the search space. We follow common practice and use short ${ }^{2}$ queries with binary relevance judgments (i.e. without "partially relevant" documents).

We consider two standard ad-hoc retrieval models to rank documents against queries: BM25 and query likelihood (QL), both implemented in the Anserini IR toolkit (Yang et al., 2017). These models use unsupervised techniques based on corpus statistics for term weighting, and will therefore be straightforwardly affected when keyphrases are added to a document. We further apply a pseudorelevance feedback method, known as RM3 (AbdulJaleel et al., 2004), on top of the models to achieve strong, near state-of-the-art retrieval results (Lin, 2019; Yang et al., 2019). For all models, we use Anserini's default parameters.

To verify the effectiveness of the adopted retrieval models, we compared their performance with that of the best participating systems in NTCIR-2. Retrieval performance is measured using mean average precision (MAP) and precision at 10 retrieved documents (P@ 10). MAP measures the overall ranking quality and $\mathrm{P} @ 10$ reflects the number of relevant documents on the first page of search results. Documents are indexed with author keywords, same as for participating systems. Results are presented in Table 1. We see that the considered retrieval models achieve strong performance, even outperforming the best participating system by a substantial margin. Note that the two best-performing systems use pseudo-relevance feedback, and that the second-ranked system is based on BM25.

\footnotetext{
${ }^{1}$ Scientific abstracts and summaries of research results.

${ }^{2}<$ description $>$ field of topic description.
}

\begin{tabular}{l|cc}
\hline Model & MAP & P@ 10 \\
\hline BM25+RM3 & $\mathbf{3 5 . 1 7}$ & $\mathbf{3 8 . 5 7}$ \\
QL+RM3 & 33.00 & 34.90 \\
$1^{\text {st }}$ (Fujita, 2001) & 31.93 & 37.35 \\
BM25 & 31.38 & 36.33 \\
$2^{\text {nd }}$ (Murata et al., 2001) & 31.31 & 36.12 \\
QL & 30.63 & 34.08 \\
$3^{\text {rd }}$ (Chen et al., 2001) & 26.24 & 33.88 \\
\hline
\end{tabular}

Table 1: Retrieval effectiveness of the considered models and the best participating systems on NTCIR-2.

\subsection{Keyphrase Generation}

Keyphrase generation is the task of producing a set of words and phrases that best summarise a document (Evans and Zhai, 1996). In contrast with most previous work that formulates this task as an extraction problem (a.k.a. keyphrase extraction), which can be seen as ranking phrases extracted from a document, recent neural models for keyphrase generation are based on sequence-to-sequence learning (Sutskever et al., 2014; Bahdanau et al., 2014), thus potentially allowing them to generate any phrase, also beyond those that appear verbatim in the text. In this study, we consider the following two neural keyphrase generation models:

seq2seq+copy (Meng et al., 2017) is a sequenceto-sequence model with attention, augmented with a copying mechanism (Gu et al., 2016) to predict phrases that rarely occur. The model is trained with document-keyphrase pairs and uses beam search decoding for inference.

seq2seq+corr (Chen et al., 2018) extends the aforementioned model with correlation constraints. It employs a coverage mechanism (Tu et al., 2016) that diversifies attention distributions to increase topic coverage, and a review mechanism to avoid generating duplicates.

We implemented the models in PyTorch (Paszke et al., 2017) using AllenNLP (Gardner et al., 2018). Models are trained on the KP20k dataset (Meng et al., 2017), which contains 567,830 scientific abstracts with gold-standard, author-assigned keywords (5.3 per doc. on avg.). We use the parameters suggested by the authors for each model.

To validate the effectiveness of our implementations, we conducted an intrinsic evaluation by counting the number of exact matches between predicted and gold keyphrases. We adopt the standard 
metric and compute the f-measure at top 5, as it corresponds to the average number of keyphrases in KP20k and NTCIR-2, that is, 5.3 and 4.8, respectively. We also examine cross-domain generalization using the KPTimes news dataset (Gallina et al., 2019), and include a state-of-the-art unsupervised keyphrase extraction model (Boudin, 2018, henceforth mp-rank) for comparison purposes. This latter baseline also provides an additional relevance signal based on graph-based ranking whose usefulness in retrieval will be tested in subsequent experiments. Results are reported in Table 2. Overall, our results are consistent with those reported in (Meng et al., 2017; Chen et al., 2018), demonstrating the superiority of well-trained neural models over unsupervised ones, and stressing their lack of robustness across domains. Rather surprisingly, seq2seq+corr is outperformed by seq2seq+copy which indicates that relevant, yet possibly redundant, keyphrases are filtered out by the added mechanisms for promoting diversity in the output.

\begin{tabular}{l|ccc}
\hline Model & KP20k & NTCIR-2 & KPTimes \\
\hline s2s+copy & $\mathbf{2 7 . 7 5}$ & $\mathbf{2 3 . 9 0}$ & $\mathbf{1 6 . 4 7}$ \\
s2s+corr & 23.78 & 22.27 & 11.73 \\
mp-rank & 14.67 & 18.10 & 14.59 \\
\hline
\end{tabular}

Table 2: f-measure at top-5 predicted keyphrases. Stemming is applied to reduce the number of mismatches.

\subsection{Extrinsic Evaluation Framework}

Our goal is to find out whether the keyphrase generation models described above are reliable enough to be beneficial for document retrieval. To do so, we contrast the performance of the retrieval models with and without automatically predicted keyphrases. Two initial indexing configurations are also examined: title and abstract only $(T+A)$, and title, abstract and author keywords $(T+A+K)$. The idea here is to investigate whether generated keyphrases simply act as a proxy for author keywords, or instead supplement them.

Unless mentioned otherwise, the top-5 predicted keyphrases are used to expand documents, which is in accordance with the average number of author keywords in NTCIR-2. We evaluate retrieval performance in terms of MAP and omit P@10 for brevity. We use the Student's paired t-test to assess statistical significance of our retrieval results at $p<0.05$ (Smucker et al., 2007).

\section{Results}

Results for retrieval models using keyphrase generation are reported in Table 3. We note that indexing keyphrases generated by seq2seq+copy, which performs best in our intrinsic evaluation, significantly improves retrieval effectiveness for all models. More interestingly, gains in effectiveness are also significant when both keyphrases and author keywords are indexed, indicating they complement each other well. This important finding suggests that predicted keyphrases are consistently helpful for document retrieval, and should be used even when author keywords are provided. Another important observation is that while both keyphrase generation models perform reasonably well in our intrinsic evaluation on NTCIR-2 (cf. Table 2, column 3), their impact on retrieval effectiveness are quite different, as only s2s+copy reaches consistent significance. This finding advocates for the importance of using document retrieval as an extrinsic evaluation task for keyphrase generation.

\begin{tabular}{l|cccc}
\hline Index & BM25 & +RM3 & QL & +RM3 \\
\hline$T+A$ & 29.16 & 31.93 & 28.98 & 31.47 \\
$\quad$ + s2s+copy & $30.54^{\dagger}$ & $\mathbf{3 4 . 3 0}$ & $30.58^{\dagger}$ & $33.26^{\dagger}$ \\
$\quad$ + s2s+corr & $30.30^{\dagger}$ & 33.24 & 29.76 & 31.38 \\
$\quad$ + mp-rank & 29.24 & 32.27 & 29.57 & 32.29 \\
\hline$T+A+K$ & 31.38 & 35.17 & 30.63 & 33.00 \\
+ s2s+copy & 31.55 & $\mathbf{3 6 . 5 3}$ & $31.70^{\ddagger}$ & $35.15^{\ddagger}$ \\
+ s2s+corr & 31.37 & 35.84 & 31.14 & 33.65 \\
$\quad$ + mp-rank & 31.38 & 35.18 & 31.23 & 33.47 \\
\hline
\end{tabular}

Table 3: MAP scores for retrieval models using various indexing configurations. $\dagger$ and $\ddagger$ indicate significance over $T+A$ and $T+A+K$, respectively.

Overall, BM25+RM3 achieves the best retrieval effectiveness, confirming previous findings on $a d$ hoc retrieval in limited data scenarios (Lin, 2019). For clarity and conciseness, we focus on this model in the rest of this paper. Encouraging diversity in keyphrases seems not to be appropriate for retrieval, as seq2seq+corr consistently gives lower results than seq2seq+copy. It is also interesting to see that the effectiveness gains of query expansion (RM3) and document expansion are additive, suggesting that they provide different but complementary relevance signals. Moreover, our results show that query expansion is more effective, which is in line with past work (Billerbeck and Zobel, 2005).

One hyper-parameter that we have deliberately left untouched so far is the number $N$ of predicted 
keyphrases that directly controls the precisionrecall trade-off of keyphrase generation models. To understand how this parameter affects retrieval effectiveness, we repeated our experiments by varying $N$ within the range $[0,9]$, and plotted the results in Figure 1. Without author keywords, we observe that all models achieve gains, but only seq2seq+copy does yield significant improvements. With author keywords, seq2seq+copy is again the only model that achieves significance, while the others show mixed results, sometimes even degrading scores. One likely explanation for this is that these models produce keyphrases that cause documents to drift away from their original meaning. We note that results are close to optimal for $N=5$, supporting our initial setting for this parameter.
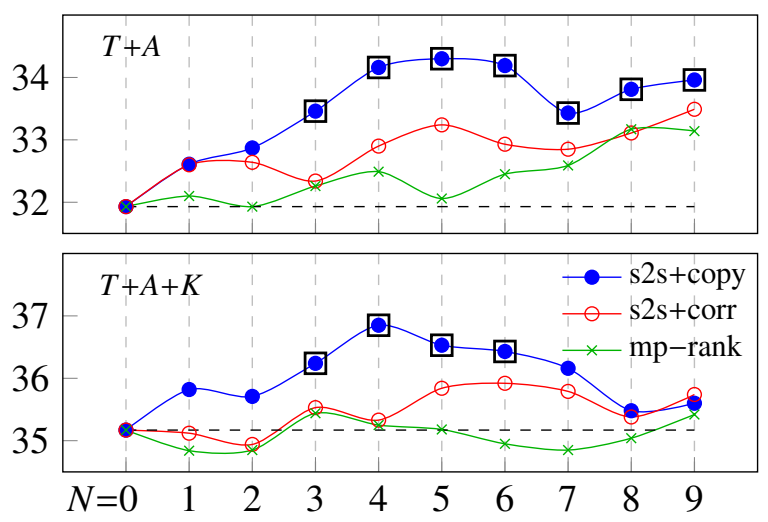

Figure 1: MAP scores for BM25+RM3 w.r.t. the number $N$ of predicted keyphrases. $\square$ denotes significance.

From our experiments, it appears that unsupervised keyphrase extraction is not effective enough to significantly improve retrieval effectiveness. The fact that keyphrase generation does so, suggests that the ability to predict absent keyphrases may be what enables better performance. Yet counterintuitively, we found that most of the gains in retrieval effectiveness are due to the high extractive accuracy of keyphrase generation models. Results in Table 4 show that expanding documents with only absent keyphrases is at best useless and at worst harmful, while using only present keyphrases brings significant improvements. We draw two conclusions from this. First, absent keyphrases may not be useful in practice unless they are tied to some form of domain terminology to prevent semantic drift. Second, as generation does not yield improvements, keyphrase extraction models may be worth further investigation. In particular, supervised models could theoretically provide similar results while being easier to train.

\begin{tabular}{l|lc|cc}
\hline \multirow{2}{*}{ Model } & \multicolumn{2}{|c|}{$T+A$ (cf. 31.93) } & \multicolumn{2}{|c}{$T+A+K$ (cf. 35.17) } \\
& pres. & abs. & pres. & abs. \\
\hline s2s+copy & $\mathbf{3 4 . 1 7}^{\dagger}$ & 32.14 & $\mathbf{3 6 . 3 0}^{\dagger}$ & 34.97 \\
s2s+corr & 32.97 & 31.96 & 36.09 & 34.77 \\
\hline
\end{tabular}

Table 4: MAP scores for BM25+RM3 using the top-5 present or absent keyphrases. $†$ indicates significance over indexing without predicted keyphrases.

Neural models for keyphrase generation exhibit a limited generalization ability, which means that their performance degrades on documents that differ from the ones encountered during training (cf. Table 2, columns 3 and 4). To quantify how much this affects retrieval effectiveness, we divided the queries into two disjoint sets: in-domain for those that belong to research fields present in KP20k, and out-domain for the others. Results are presented in Table 5. The first thing we notice is the overall lower performance of out-domain queries, which may be explained by the unbalanced distribution of domains in the NTCIR-2 collection. Most importantly, out-domain queries on full indexing (i.e. $T+A+K$ ) is the only configuration in which no significant gains in retrieval effectiveness are achieved. This last experiment shows that expanding documents using existing keyphrase generation models may be ineffective in the absence of in-domain training data, and stresses the need of domain adaptation for keyphrase generation.

\begin{tabular}{l|rr|rc}
\hline \multirow{2}{*}{ Model } & \multicolumn{2}{|c|}{$T+A$} & \multicolumn{2}{c}{$T+A+K$} \\
& $\mathbf{I}(32.70)$ & $\mathbf{O}(30.99)$ & $\mathbf{I}(36.18)$ & $\mathbf{O}(33.93)$ \\
\hline s2s+copy & $\mathbf{3 5 . 4 0}^{\dagger}$ & $\mathbf{3 2 . 9 6}^{\dagger}$ & $\mathbf{3 8 . 1 3}$ & $\mathbf{3 4 . 5 5}$ \\
s2s+corr & 33.49 & 32.92 & 37.13 & 34.25 \\
mp-rank & 32.73 & 31.71 & 36.74 & 33.26 \\
\hline
\end{tabular}

Table 5: MAP scores for BM25+RM3 on in-domain (I) and out-domain $(\mathrm{O})$ queries. $\dagger$ indicates significance over w/o keyphrases whose scores are in parentheses.

\section{Conclusion}

We presented the first study of the usefulness of keyphrase generation for scientific document retrieval. Our results show that keyphrases can significantly improve retrieval effectiveness, and also highlight the importance of evaluating keyphrase generation models from an extrinsic perspective. Other retrieval tasks may also benefit from using keyphrase information and we expect our results to serve as a basis for further improvements. 


\section{Acknowledgements}

We thank the anonymous reviewers for their valuable comments. This work was supported by the IKEBANA project (grant of Atlanstic 2020) and the French National Research Agency (ANR) through the DELICES project (ANR-19-CE38-0005-01).

\section{References}

Nasreen Abdul-Jaleel, James Allan, W Bruce Croft, Fernando Diaz, Leah Larkey, Xiaoyan Li, Mark D Smucker, and Courtney Wade. 2004. Umass at trec 2004: Novelty and hard. Computer Science Department Faculty Publication Series, page 189.

Alan R Aronson, James G Mork, Clifford W Gay, Susanne M Humphrey, and Willie J Rogers. 2004. The nlm indexing initiative's medical text indexer. In Medinfo, pages 268-272.

Dzmitry Bahdanau, Kyunghyun Cho, and Yoshua Bengio. 2014. Neural machine translation by jointly learning to align and translate. arXiv preprint arXiv:1409.0473.

Frances H Barker, Douglas C Veal, and Barry K Wyatt. 1972. Comparative efficiency of searching titles, abstracts, and index terms in a free-text data base. Journal of Documentation, 28(1):22-36.

Bodo Billerbeck and Justin Zobel. 2005. Document expansion versus query expansion for ad-hoc retrieval. In Proceedings of the 10th Australasian Document Computing Symposium, pages 34-41. Citeseer.

Lutz Bornmann and Rüdiger Mutz. 2015. Growth rates of modern science: A bibliometric analysis based on the number of publications and cited references. Journal of the Association for Information Science and Technology, 66(11):2215-2222.

Florian Boudin. 2018. Unsupervised keyphrase extraction with multipartite graphs. In Proceedings of the 2018 Conference of the North American Chapter of the Association for Computational Linguistics: $\mathrm{Hu}$ man Language Technologies, Volume 2 (Short Papers), pages 667-672, New Orleans, Louisiana. Association for Computational Linguistics.

Adrien Bougouin, Florian Boudin, and Béatrice Daille. 2013. Topicrank: Graph-based topic ranking for keyphrase extraction. In Proceedings of the Sixth International Joint Conference on Natural Language Processing, pages 543-551, Nagoya, Japan. Asian Federation of Natural Language Processing.

Adrien Bougouin, Florian Boudin, and Béatrice Daille. 2016. Keyphrase annotation with graph co-ranking. In Proceedings of COLING 2016, the 26th International Conference on Computational Linguistics: Technical Papers, pages 2945-2955, Osaka, Japan. The COLING 2016 Organizing Committee.
Aitao Chen, Fredric C Gey, and Hailing Jiang. 2001. Berkeley at ntcir-2: Chinese, japanese, and english ir experiments. In Proceedings of the Second NTCIR Workshop on Research in Chinese $\mathcal{F}$ Japanese Text Retrieval and Text Summarization.

Jun Chen, Xiaoming Zhang, Yu Wu, Zhao Yan, and Zhoujun Li. 2018. Keyphrase generation with correlation constraints. In Proceedings of the 2018 Conference on Empirical Methods in Natural Language Processing, pages 4057-4066, Brussels, Belgium. Association for Computational Linguistics.

Miles Efron, Peter Organisciak, and Katrina Fenlon. 2012. Improving retrieval of short texts through document expansion. In Proceedings of the 35th International ACM SIGIR Conference on Research and Development in Information Retrieval, SIGIR '12, pages 911-920, New York, NY, USA. ACM.

David A. Evans and Chengxiang Zhai. 1996. Noun phrase analysis in large unrestricted text for information retrieval. In Proceedings of the 34th Annual Meeting of the Association for Computational Linguistics, pages 17-24, Santa Cruz, California, USA. Association for Computational Linguistics.

Corina Florescu and Cornelia Caragea. 2017. Positionrank: An unsupervised approach to keyphrase extraction from scholarly documents. In Proceedings of the 55th Annual Meeting of the Association for Computational Linguistics (Volume 1: Long Papers), pages 1105-1115, Vancouver, Canada. Association for Computational Linguistics.

Sumio Fujita. 2001. Notes on the limits of clir effectiveness: Ntcir-2 evaluation experiments at justsystem. In Proceedings of the Second NTCIR Workshop on Research in Chinese $\mathcal{F}$ Japanese Text Retrieval and Text Summarization.

Ygor Gallina, Florian Boudin, and Béatrice Daille. 2019. Kptimes: A large-scale dataset for news keyphrase generation. In Proceedings of the 12th International Conference on Natural Language Generation. Association for Computational Linguistics.

Matt Gardner, Joel Grus, Mark Neumann, Oyvind Tafjord, Pradeep Dasigi, Nelson F. Liu, Matthew Peters, Michael Schmitz, and Luke Zettlemoyer. 2018. AllenNLP: A deep semantic natural language processing platform. In Proceedings of Workshop for NLP Open Source Software (NLP-OSS), pages 16, Melbourne, Australia. Association for Computational Linguistics.

Walter Greulich. 2011. Scientific texts and the indexer. The Indexer: The International Journal of Indexing, 29(3):114-122.

Jiatao Gu, Zhengdong Lu, Hang Li, and Victor O.K. Li. 2016. Incorporating copying mechanism in sequence-to-sequence learning. In Proceedings of the 54th Annual Meeting of the Association for Computational Linguistics (Volume 1: Long Papers), 
pages 1631-1640, Berlin, Germany. Association for Computational Linguistics.

Carl Gutwin, Gordon Paynter, Ian Witten, Craig NevillManning, and Eibe Frank. 1999. Improving browsing in digital libraries with keyphrase indexes. Decis. Support Syst., 27(1-2):81-104.

Kazi Saidul Hasan and Vincent Ng. 2014. Automatic keyphrase extraction: A survey of the state of the art. In Proceedings of the 52nd Annual Meeting of the Association for Computational Linguistics (Volume 1: Long Papers), pages 1262-1273, Baltimore, Maryland. Association for Computational Linguistics.

Xin Jiang, Yunhua Hu, and Hang Li. 2009. A ranking approach to keyphrase extraction. In Proceedings of the 32Nd International ACM SIGIR Conference on Research and Development in Information Retrieval, SIGIR '09, pages 756-757, New York, NY, USA. ACM.

Noriko Kando. 2001. Overview of the second ntcir workshop. In Proceedings of the Second NTCIR Workshop on Research in Chinese $\mathcal{E}$ Japanese Text Retrieval and Text Summarization.

Jimmy Lin. 2019. The neural hype and comparisons against weak baselines. SIGIR Forum, 52(2):40-51.

Ke Liu, Shengwen Peng, Junqiu Wu, Chengxiang Zhai, Hiroshi Mamitsuka, and Shanfeng Zhu. 2015. Meshlabeler: improving the accuracy of large-scale mesh indexing by integrating diverse evidence. Bioinformatics, 31(12):i339-i347.

Zhiyuan Liu, Wenyi Huang, Yabin Zheng, and Maosong Sun. 2010. Automatic keyphrase extraction via topic decomposition. In Proceedings of the 2010 Conference on Empirical Methods in Natural Language Processing, pages 366-376, Cambridge, MA. Association for Computational Linguistics.

Kun Lu and Margaret E.I. Kipp. 2014. Understanding the retrieval effectiveness of collaborative tags and author keywords in different retrieval environments: An experimental study on medical collections. Journal of the Association for Information Science and Technology, 65(3):483-500.

Rui Meng, Sanqiang Zhao, Shuguang Han, Daqing He, Peter Brusilovsky, and Yu Chi. 2017. Deep keyphrase generation. In Proceedings of the 55th Annual Meeting of the Association for Computational Linguistics (Volume 1: Long Papers), pages 582-592, Vancouver, Canada. Association for Computational Linguistics.

Rada Mihalcea and Paul Tarau. 2004. Textrank: Bringing order into texts. In Proceedings of EMNLP 2004, pages 404-411, Barcelona, Spain. Association for Computational Linguistics.
Masaki Murata, Masao Utiyama, Qing Ma, Hiromi Ozaku, and Hitoshi Isahara. 2001. Crl at ntcir2. In Proceedings of the Second NTCIR Workshop on Research in Chinese $\mathcal{E}$ Japanese Text Retrieval and Text Summarization.

Thuy Dung Nguyen and Min-Yen Kan. 2007. Keyphrase extraction in scientific publications. In Proceedings of the 10th International Conference on Asian Digital Libraries: Looking Back 10 Years and Forging New Frontiers, ICADL'07, pages 317-326, Berlin, Heidelberg. Springer-Verlag.

Rodrigo Nogueira, Wei Yang, Jimmy Lin, and Kyunghyun Cho. 2019. Document expansion by query prediction. arXiv preprint arXiv:1904.08375.

Adam Paszke, Sam Gross, Soumith Chintala, Gregory Chanan, Edward Yang, Zachary DeVito, Zeming Lin, Alban Desmaison, Luca Antiga, and Adam Lerer. 2017. Automatic differentiation in pytorch. In NIPS 2017 Workshop Autodiff.

Shengwen Peng, Ronghui You, Hongning Wang, Chengxiang Zhai, Hiroshi Mamitsuka, and Shanfeng Zhu. 2016. Deepmesh: deep semantic representation for improving large-scale mesh indexing. Bioinformatics, 32(12):i70-i79.

Mark D. Smucker, James Allan, and Ben Carterette. 2007. A comparison of statistical significance tests for information retrieval evaluation. In Proceedings of the Sixteenth ACM Conference on Conference on Information and Knowledge Management, CIKM '07, pages 623-632, New York, NY, USA. ACM.

C Rockelle Strader. 2011. Author-assigned keywords versus library of congress subject headings. Library resources $\mathcal{E}$ technical services, 53(4):243-250.

Ilya Sutskever, Oriol Vinyals, and Quoc V Le. 2014. Sequence to sequence learning with neural networks. In Z. Ghahramani, M. Welling, C. Cortes, N. D. Lawrence, and K. Q. Weinberger, editors, Advances in Neural Information Processing Systems 27, pages 3104-3112. Curran Associates, Inc.

Takafumi Suzuki, Kiyoko Uchiyama, Ryota Tomisaka, and Akiko Aizawa. 2011. Analyzing the characteristics of academic paper categories by using an index of representativeness. In Proceedings of the 25th Pacific Asia Conference on Language, Information and Computation, pages 587-596, Singapore. Institute of Digital Enhancement of Cognitive Processing, Waseda University.

Tao Tao, Xuanhui Wang, Qiaozhu Mei, and ChengXiang Zhai. 2006. Language model information retrieval with document expansion. In Proceedings of the Human Language Technology Conference of the NAACL, Main Conference, pages 407-414, New York City, USA. Association for Computational Linguistics. 
Zhaopeng Tu, Zhengdong Lu, Yang Liu, Xiaohua Liu, and Hang Li. 2016. Modeling coverage for neural machine translation. In Proceedings of the 54th Annual Meeting of the Association for Computational Linguistics (Volume 1: Long Papers), pages 7685, Berlin, Germany. Association for Computational Linguistics.

Peter D. Turney. 2003. Coherent keyphrase extraction via web mining. In Proceedings of the 18th International Joint Conference on Artificial Intelligence, IJCAI'03, pages 434-439, San Francisco, CA, USA. Morgan Kaufmann Publishers Inc.

Xiaojun Wan and Jianguo Xiao. 2008. Collabrank: Towards a collaborative approach to single-document keyphrase extraction. In Proceedings of the 22nd International Conference on Computational Linguistics (Coling 2008), pages 969-976, Manchester, UK. Coling 2008 Organizing Committee.

I. H. Witten and O. Medelyan. 2006. Thesaurus based automatic keyphrase indexing. In Proceedings of the 6th ACM/IEEE-CS Joint Conference on Digital Libraries (JCDL '06), pages 296-297.

Ian H. Witten, Gordon W. Paynter, Eibe Frank, Carl Gutwin, and Craig G. Nevill-Manning. 1999. Kea: Practical automatic keyphrase extraction. In Proceedings of the Fourth ACM Conference on Digital Libraries, DL '99, pages 254-255, New York, NY, USA. ACM.

Peilin Yang, Hui Fang, and Jimmy Lin. 2017. Anserini: Enabling the use of lucene for information retrieval research. In Proceedings of the 40th International ACM SIGIR Conference on Research and Development in Information Retrieval, SIGIR '17, pages 1253-1256, New York, NY, USA. ACM.

Wei Yang, Kuang Lu, Peilin Yang, and Jimmy Lin. 2019. Critically examining the "neural hype": Weak baselines and the additivity of effectiveness gains from neural ranking models. In Proceedings of the $42 N d$ International ACM SIGIR Conference on Research and Development in Information Retrieval, SIGIR'19, pages 1129-1132, New York, NY, USA ACM.

Chengxiang Zhai. 1997. Fast statistical parsing of noun phrases for document indexing. In Fifth Conference on Applied Natural Language Processing, pages 312-319, Washington, DC, USA. Association for Computational Linguistics.

Jing Zhao and Yuxiang Zhang. 2019. Incorporating linguistic constraints into keyphrase generation. In Proceedings of the 57th Annual Meeting of the Association for Computational Linguistics, pages 5224-5233, Florence, Italy. Association for Computational Linguistics. 


\section{A Supplementary material}

\section{A.1 Related Work}

\section{Keyphrase extraction and generation}

Identifying keyphrases for a given document is a long standing task in NLP. Earlier work typically involves two steps: 1) extracting keyphrase candidates; and 2) ranking those candidates by importance. Models mainly differ in how they do the latter, commonly used techniques being supervised learning (Witten et al., 1999; Turney, 2003; Nguyen and Kan, 2007; Jiang et al., 2009) and graph-based methods (Mihalcea and Tarau, 2004; Wan and Xiao, 2008; Bougouin et al., 2013; Florescu and Caragea, 2017). These models are, however, inherently limited in the sense that they can only output keyphrases that appear in the text. To allow the prediction of keyphrases describing implicit topics or using different wordings, previous work relied on external resources like controlled vocabularies (Witten and Medelyan, 2006; Bougouin et al., 2016), while recent attempts leveraged neural generative models (Meng et al., 2017; Chen et al., 2018; Zhao and Zhang, 2019).

\section{Biomedical indexing}

Also related to our work is the research done on biomedical semantic indexing using $\mathrm{MeSH}^{3}$, a hierarchically-organized controlled vocabulary. Automated methods for assigning MeSH terms make use of all sorts of techniques, such as pattern matching (Aronson et al., 2004) or learning to rank (Liu et al., 2015; Peng et al., 2016).

\section{Document expansion}

Our work is similar in nature to previous research on document expansion (Tao et al., 2006; Efron et al., 2012), and is closely related to recent work on document expansion using automatically generated queries (Nogueira et al., 2019).

\section{A.2 Parameters}

Table 6 displays the model parameters we use for seq2seq+copy and seq2seq+corr.

Table 7 presents the research fields used for dividing queries into two sets.

\section{A.3 Example}

An example of document along with automatically generated keyphrases is shown in Table 8.

\begin{tabular}{l|c}
\hline Parameter & Value \\
\hline Network & bi-GRU \\
Vocabulary size & $50 \mathrm{~K}$ \\
Word embedding size & 150 \\
Hidden layer & 2 \\
Hidden layer size & 300 \\
Optimizer & Adam \\
Initial learning rate & $10^{-4}$ \\
Gradient clipping & 0.1 \\
Dropout & 0.5 \\
Beam depth & 6 \\
Beam size & 200 \\
\hline
\end{tabular}

Table 6: Model Parameters.

\begin{tabular}{|c|c|c|}
\hline Research field & In & Out \\
\hline Electricity, information and control & $\checkmark$ & - \\
\hline Chemistry & $\checkmark$ & - \\
\hline Architecture, civil engineering & - & $\checkmark$ \\
\hline Biology and agriculture & - & $\checkmark$ \\
\hline Science & $\checkmark$ & - \\
\hline Engineering & $\checkmark$ & - \\
\hline Medicine and dentistry & - & $\checkmark$ \\
\hline Cultural and social science & - & $\checkmark$ \\
\hline \# of queries & 27 & 22 \\
\hline
\end{tabular}

Table 7: Research fields for in- and out-domain queries.

\footnotetext{
${ }^{3}$ https://www.nlm.nih.gov/mesh/
} 
title Grammatical Inference for Concept Acquisition from Documents.

abstract The purpose of this study is to acquire knowledge from large scale natural language documents. There are two types of knowledge in the documents. One is explicitly represented knowledge which is acquired using natural language processing. The other is implicit constrain. In this paper, how to acquire implicit constraint using grammatical inference from the documents is described. We propose a grammatical inference system which uses inference rules based on logic, and show that the system can learn easy pattern of character lists. We also discuss its application to knowledge acquisition from real documents.

\begin{aligned} & \hline gold $\begin{array}{l}\text { grammatical inference // knowledge acquisition // logic progamming // concept } \\ \text { learning }\end{array} \\ &$\hline s2s+copy $\begin{array}{l}\text { grammatical inference // knowledge // grammatical // knowledge representation // } \\ \text { natural language processing }\end{array} \\ &$\hline s2s+corr $\begin{array}{l}\text { grammatical inference // knowledge acquisition // concept acquisition // inference } \\ \text { rules // natural language processing }\end{array} \\ &$\hline mp-rank grammatical inference // documents // knowledge // large scale // concept acquisition \\ & \hline\end{aligned}

Table 8: Example document (id: gakkai-e-0001014453 from ntc2-e1g) with author keywords (gold) and automatically generated keyphrases. We note a typo in the gold annotation (progamming). 